\title{
A real-time vehicle-terramechanics model for visual simulation
}

\author{
SUN Dong-bo, DI Yan-qiang, CUI Hao-hao
}

Ordnance Engineering College , Shijiazhuang 050003, China;

\begin{abstract}
Keywords: terramechanics; Wheel-terrain interaction; visual simulation.
\end{abstract}
\begin{abstract}
The vehicle-terrain interaction model plays a critical role in off-road vehicle driving visual simulations. This paper present a new terrain deformation model for simulating vehicle-terrain interaction. The model simplifies the Bekker's classic terramechanics model, and calculates terrain deformation according to the vehicle load, velocity, tire size, and soil concentration in real time. Simulation results show that the simplified terramechanics model is credible and real-time. This method can simulate vehicles on common types of soft terrains such as clay, sand, and be used in visual simulation.
\end{abstract}

\section{Introduction}

Off-road vehicle (including wheeled and tracked) has a good passing ability and been widely used in the modern military, agriculture, construction and other fields. But the driver training for the vehicle mostly uses real vehicles, that is low efficiency, high power consumption, pollution of the environment, long training period, so more and more vehicle driving simulators are developed and utilized. But most vehicle driving simulators is developed by visual simulation software such as OpenGVS, Vega Prime, Unity3D ${ }^{[1]}$. There are still many problems, such as lack of real-time dynamic interaction between wheel and ground, lack of real-time terrain deformation, lack of immersion and realism. And most vehicle simulators only make models for road and other man-made road surface, and not consider the condition that vehicles drive on off-road. In addition, as the development of vehicle-terramechanics and multi-body dynamics ${ }^{[2][3]}$, domestic and foreign scholars have done a lot of research on vehicle dynamics. By establishing completed and detailed vehicle model in DADS, ADAMS / ATV, RecurDyn and other multi-body simulation software, the researchers can simulate the dynamic behaviors in the real driving process ${ }^{[4][5]}$. But a few more degrees of freedom, system complexity, dynamic response slow, the calculation speed not achieving the real-time requirements of visual simulation, so that models are not conducive to the development of visual simulation program. Although these are quite good research for virtual reality simulation technology and vehicle-terramechanics in domestic and foreign, the development work effectively combining of both in a real-time interactive simulation platform still needs to be further deepened.

To solve the above problems, by analyzing the interactive force between driven wheels and soft terrain, on the basis of Bekker's pressure model, Wong's pressure theory and Janosi's shear theory the vehicle-terramechanics semi-empirical model has been simplified and the simplified model keep authenticity and real-time. So that it can be applied to virtual driving off-road vehicles and other visual simulation fields.

\section{The Vehicle-terramechanics Semi-empirical Model}

The typical representative of vehicle-terramechanics semi-empirical method is Bekker's study. This method base the classical soil mechanics (such as the doctrine of Coulomb, Terzaghi, Rankin and Plante) and present a series of semi-empirical soil-vehicle system engineering model (formula) through a large number of laboratory simulation experiments[2].Semi-empirical model characterize the physical properties of terrain. The relationship between track and terrain can be represented by a semi-empirical and provides a theoretical basis for vehicle's performance prediction. This method has been the most applications in vehicle-terramechanics research. The model can calculate the wheel 
sinkage, the horizontal force, torque and so on, after knowing the parameters of terrain and wheel vertical load.

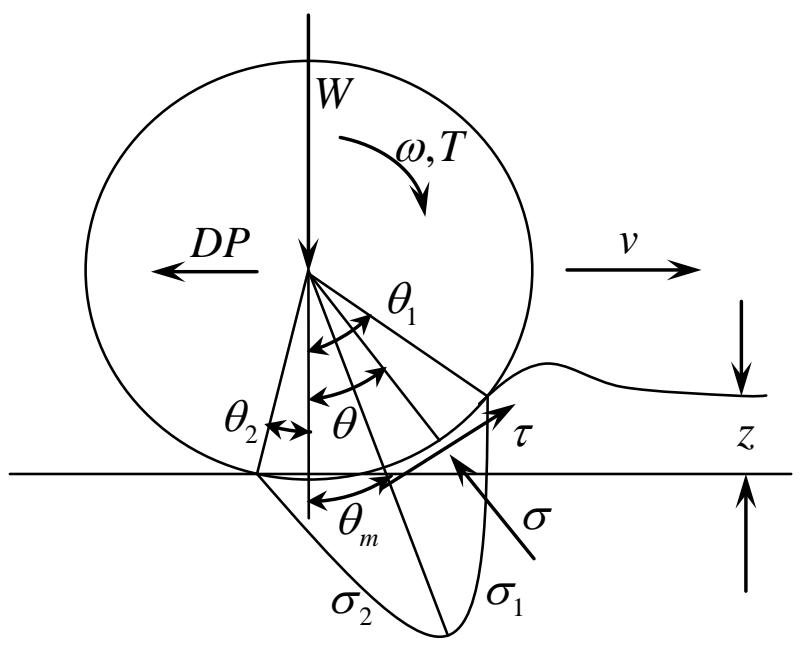

Fig.1. Free-body diagram of a rigid wheel on deformable terrain.

The interaction force analysis between driven wheels and soft terrain is shown in Fig. 1. The interaction force performance as continuous normal stress $\sigma$ and shear stress $\tau$.

According to Bekker's pressure model[2] and Wong's pressure theory[6][7], the formula of normal stress as follows:

$$
\begin{aligned}
& \sigma_{1}(\theta)=\left(\frac{\mathrm{k}_{c}}{b}+\mathrm{k}_{\phi}\right) \cdot\left(\mathrm{r}\left(\cos \theta-\cos \theta_{1}\right)\right)^{n} \\
& \sigma_{2}(\theta)=\left(\frac{\mathrm{k}_{c}}{b}+\mathrm{k}_{\phi}\right) \cdot \\
& \left(\mathrm{r}\left(\cos \left(\theta_{1}-\frac{\theta-\theta_{2}}{\theta_{m}-\theta_{2}}\left(\theta_{1}-\theta_{m}\right)\right)-\cos \theta_{1}\right)\right)^{n}
\end{aligned}
$$

where $k_{c}$ and $k_{\phi}$ are the soil cohesion frictional constants, $b$ is the wheel width, $r$ is the wheel radius, $n$ is the sinkage exponent, $\theta_{1}$ is the angle from the vertical at which the wheel first makes contact with the terrain, $\theta_{2}$ is the angle from the vertical at which the wheel loses contact with the terrain, a typical assumption is that the soil has no rebound, thus $\theta_{2}=0$, and $\theta_{m}$ is the angle from the vertical at which the maximum stress occurs.

Shear stress is derived from the Janosi-Hanamoto shear slip/stress empirical relationship[8].

$$
\tau=(\mathrm{c}+\sigma \tan \phi)\left(1-\mathrm{e}^{-j / k}\right)
$$

where $c$ is the soil shear cohesion constant, $\phi$ is the angle of internal friction, $\sigma$ is the normal pressure at the point, $k$ is the shear deformation modulus, and $j$ is the slip displacement. The shear deformation was described according to Bekker in wheel coordinates as:

$$
j=\int_{0}^{t} v_{j} d t=r\left[\theta_{1}-\theta-(1-\mathrm{i})\left(\sin \theta_{1}-\sin \theta\right)\right]
$$

Force balance equations for the system in Fig. 1 can be written as:

$$
\begin{gathered}
W=r b\left(\int_{\theta_{2}}^{\theta_{1}} \sigma(\theta) \cos \theta d \theta+\int_{\theta_{2}}^{\theta_{1}} \tau(\theta) \sin \theta d \theta\right) \\
D P=r b\left(\int_{\theta_{2}}^{\theta_{1}} \tau(\theta) \cos \theta d \theta-\int_{\theta_{2}}^{\theta_{1}} \sigma(\theta) \sin \theta d \theta\right) \\
T=r^{2} b \int_{\theta_{2}}^{\theta_{1}} \tau(\theta) d \theta
\end{gathered}
$$

Substituting(1), (2), (3) into (4) and according the vertical load $W$ and the wheel slip $i$, we can compute out the angle $\theta_{1}$, the maximum values of the normal stress angle $\theta_{m}$ and the wheel sinkage 
$z$ by computer iteration method[9]. Further according to the equation (5) and (6), we can compute out the horizontal force $D P$ and the torque $T$.

\section{Vehicle-terramechanics Model Simplification}

Integration and iterative process exists in the above model, so that the calculation speed can not achieve real-time requirement for the visual simulation. Therefore, we simplify semi-empirical model to increase its calculation speed

Fig. 2 shows typical simulated plots of shear and normal stress distributions (as defined by (1), (2), and (3), respectively) around the rim of a driven rigid wheel on various terrains at moderate wheel slip $(i=0.1)$. Parameters used in these plots are listed in Table 1 and represent a diverse range of terrain types: dry sand, sandy loam, and clayey soil. While the simulated wheel radius $r$ was $0.2 \mathrm{~m}$, and the wheel width $b$ was $0.1 \mathrm{~m}$. The simulation results show that the shear and normal stress distribution curves are approximately linear for a diverse range of terrains and the locations of maximum values of the normal and shear stress are very close.

Fig. 3 shows shat simulated plots of shear and normal stress distributions around the rim of a driven rigid wheel on dry sand terrains at moderate wheel $\operatorname{slip}(i=0.1)$ in different entry angle. While the simulated wheel radius $r$ was $0.2 \mathrm{~m}$, and the wheel width $b$ was $0.1 \mathrm{~m}$. The simulation results show that with increasing the entry angle, the stress increase corresponding, but the stress distributions trend are similar

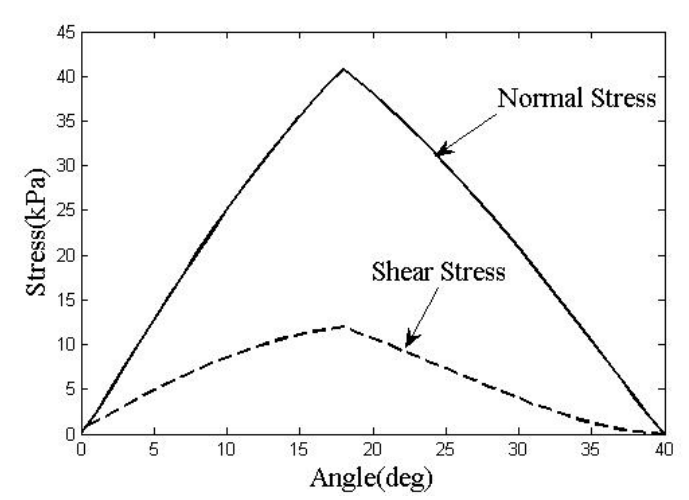

a ) Dry Sand

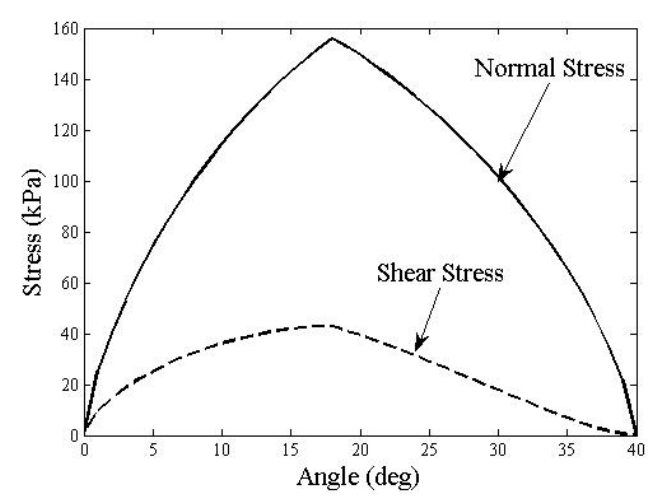

b) Sandy loam

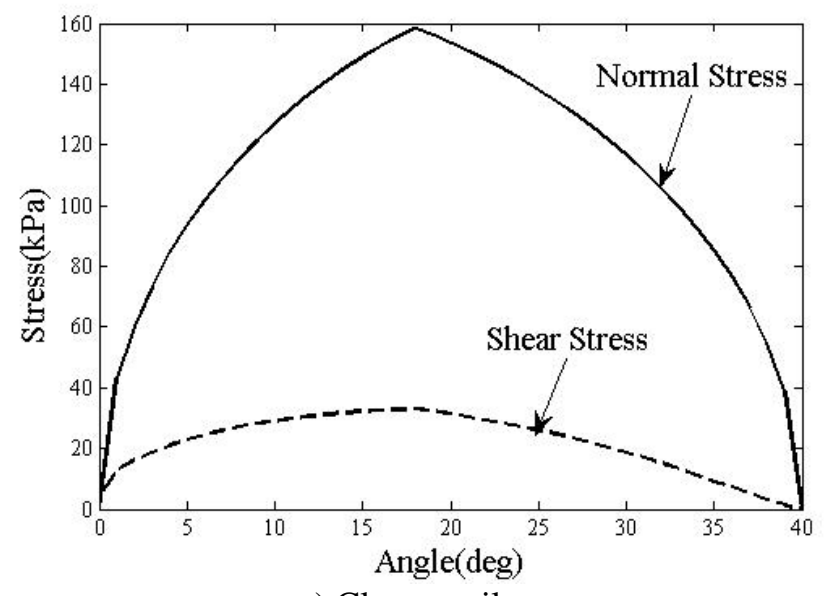

c) Clayey soil

Fig. 2. Normal and shear stress distribution for various terrain types at moderate wheel slip. 


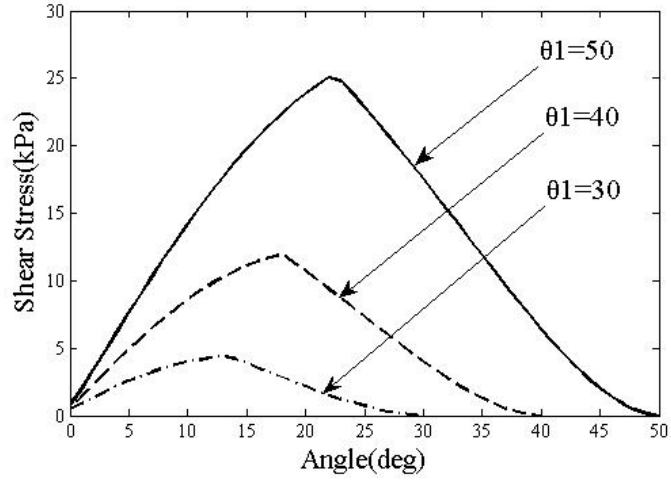

a) Normal stress distribution

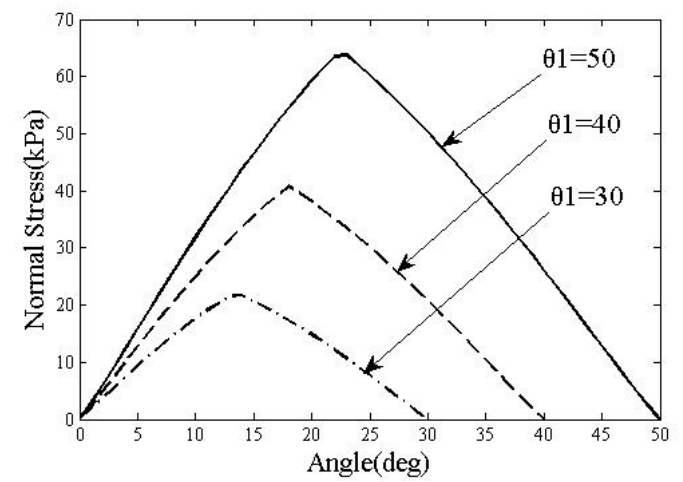

b) Shear stress distribution

Fig. 3. Normal and shear stress distribution for different entry angle.

According to the stress distribution of the wheel, we propose the following assumptions to simplify the vehicle-terramechanics model:

a) the exit angle $\theta_{2}$ is 0 ;

b) the normal stress and shear stress distribution is approximately linear;

c) The maximum normal stress and maximum shear stress at the same point, namely at $\theta_{m}[10]$;

According to the assumption, simplified stress distribution linear formula as follows:

$$
\begin{gathered}
\sigma_{1}(\theta)=\frac{\theta_{1}-\theta}{\theta_{1}-\theta_{m}} \sigma_{m} \\
\sigma_{2}(\theta)=\frac{\theta}{\theta_{m}} \sigma_{m} \\
\tau_{1}(\theta)=\frac{\theta_{1}-\theta}{\theta_{1}-\theta_{m}} \tau_{m} \\
\tau_{2}(\theta)=c+\frac{\theta}{\theta_{m}}\left(\tau_{m}-\mathrm{c}\right)
\end{gathered}
$$

where $\sigma_{m}$ and $\tau_{m}$ are the maximum values of the normal and shear stress:

$$
\begin{gathered}
\sigma_{m}=\left(\frac{\mathrm{k}_{c}}{b}+\mathrm{k}_{\phi}\right)\left(\mathrm{r}\left(\cos \theta_{m}-\cos \theta_{1}\right)\right)^{n} \\
\tau_{m}=\left(\mathrm{c}+\sigma_{m} \tan \phi\right)\left(1-\mathrm{e}^{-r\left[\theta_{1}-\theta_{m}-(1-i)\left(\sin \theta_{1}-\sin _{m}\right)\right] / k}\right)
\end{gathered}
$$

Substituting (7) (12) into (4) yields expressions for the vertical load $W$ :

$$
\begin{aligned}
& W=\frac{r b}{\theta_{m}\left(\theta_{1}-\theta_{m}\right)}\left[\tau_{m}\left(\theta_{1} \sin \theta_{m}-\theta_{m} \sin \theta_{1}\right)\right. \\
& +\sigma_{m}\left(\theta_{1} \cos \theta_{m}-\theta_{m} \cos \theta_{1}-\theta_{1}+\theta_{m}\right) \\
& \left.+c\left(\theta_{m} \sin \theta_{m}-\theta_{1} \sin \theta_{m}+\theta_{1} \theta_{m}-\theta_{m}^{2}\right)\right]
\end{aligned}
$$

According to the formula (13), we can compute the entry angle $\theta_{1}$ and wheel sinkage $z$ by iterative computer method under knowing the characteristic parameters of terrain, vertical load $W$, and wheel slip $i$. Substituting the entry angle $\theta_{1}$ into (5) (6) yields the horizontal force $D P$ and the torque $T$ separately.

Although according to equation (13) we can not solve the wheel sinkage $Z$ corresponding to the vertical load $W$ and wheel slip $i$, the computing part eliminates the integral part in equation (4) and the speed of compute iteration greatly accelerates. The simplified model can compute the wheel sinkage $Z$ according the vertical load $W$ and wheel slip $i$ in real-time. So that it can meet the real-time requirements for visual simulation. 
Table 1 Parameters for various terrain types

\begin{tabular}{llll}
\hline & Dry sand & Sandy loam & Clayey soil \\
\hline$n$ & 1.1 & 0.7 & 0.5 \\
$c(\mathrm{kPa})$ & 1.0 & 1.7 & 4.14 \\
$\phi(\mathrm{deg})$ & 30.0 & 29.0 & 13.0 \\
$k_{c}\left(\mathrm{kPa} / \mathrm{m}^{n-1}\right)$ & 0.9 & 5.3 & 13.2 \\
$k_{\phi}\left(\mathrm{kPa} / \mathrm{m}^{n}\right)$ & 1523.4 & 1515.0 & 692.2 \\
$k(\mathrm{~m})$ & 0.025 & 0.025 & 0.01 \\
\hline
\end{tabular}

\section{The Simulation Results}

We simulate and compare the vehicle-terramechanics semi-empirical model and the simplified one. The results show in Fig. 4, Fig. 5. Where the terrain type is dry sand in table 1, the wheel width is $0.1 \mathrm{~m}$, and the wheel radius is $0.2 \mathrm{~m}$. Fig. 4 shows that the relationship between the wheel sinkage $z$ and wheel slip $i$ under three vertical load cases $W=100 N 、 W=200 N 、 W=300 N$. The simulation results indicate that the distinction between the wheel sinkage theoretical and simplified value is maximum at wheel slip $i=0$, decreases with the wheel slip $i$ decreasing. And it is less than $10 \%$ and decreases with vertical load increasing. Fig. 5 shows that the relationship between the horizontal force $D P$ and wheel slip $i$ under three load cases $W=100 N 、 W=200 N 、 W=300 N$. The simulation results indicate that the distinction between the horizontal force theoretical and simplified value is less than $5 \%$ The simulation results show that the simplified model have a good credibility.

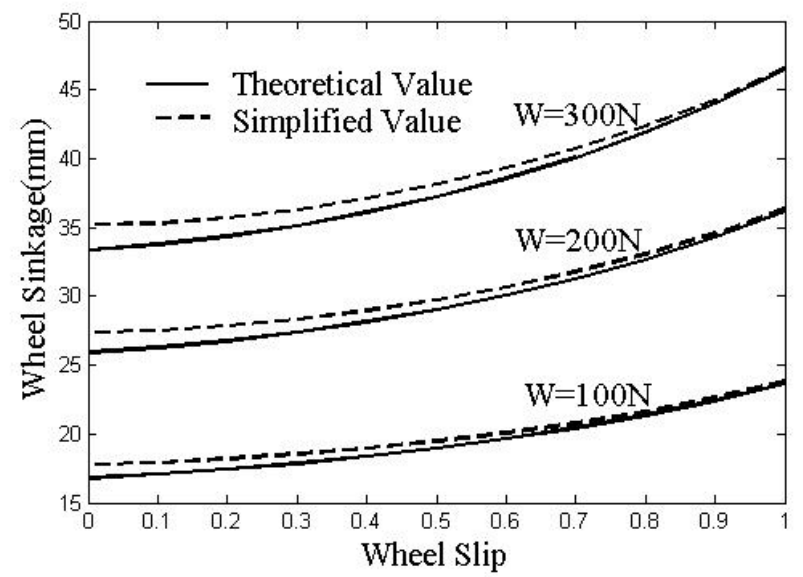

Fig. 4. Relationship between wheel sinkage and wheel slip

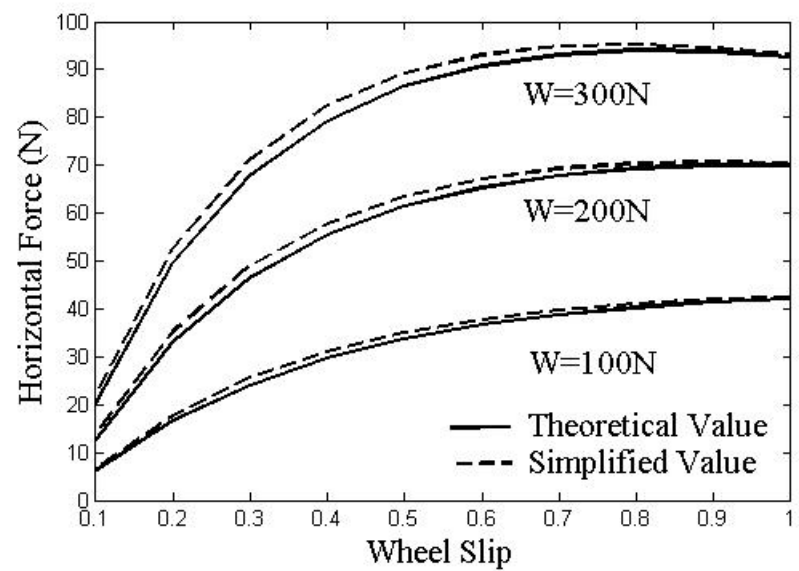

Fig. 5. Relationship between horizonal force and wheel slip 


\section{Summary}

In this paper, we simplify the vehicle-terramechanics semi-empirical model and compare the theoretical and simplified value of wheel sinkage, horizontal force and so on. The simulation results indicate that the simplified model is credible and real-time. So that it can be applied to the field of off-road vehicles in the virtual driving training.

\section{References}

[1] Su Mingming. The Visualized Virtual Simulation of Hydraulic Excavator Based on Multigen Creator/Vega Prime [D]. Southwest Jiaotong University, 2011.

[2] Bekker M G. Theory of Land Locomotion[M]. The Univ. of Michigan press, 2001.

[3] Okada Y, Nagatani K, Yoshida K, et al. Shared autonomy system for tracked vehicles on rough terrain based on continuous three-dimensional terrain scanning[J]. Journal of Field Robotics, 2011, 28(6):875-893.

[4] LUO Qing-guo, SI Dong-ya, GONG Zheng-bo. Dynamic Simulation of a Tracked Vehicle Based on RecurDyn[J]. Vehicle and Power Technology, 2011, 43(9):42-45.

[5] Chen Yuanyuan. The Dynamic Analysis and Simulation of Tracked Vehicles[D]. Shenyang Ligong University, 2011.

[6] Wong J Y. Theory of Ground Vehicle[M]. John Wiley, 1978.

[7] Reece A R. , Wong J K. Soil Failure Beneath Rigid Wheels[C]. Proc. Second Int. Conf. of ISTVS, Quebec, 1966.

[8] Z. Janosi, Hanamoto B. Analytical Determination of Drawbar Pull as a Function of Slip for Tracked Vehicle in Deformable Soils[C]. Proc. 1st Int. Conf. of ISTVS, Torino. 1961: 707-736.

[9] Fan Huiwen. Terrain-vehicles system model and performance predication[J]. Equipment for Geophysical Prospecting, 1997,7(4):7-12.

[10] Iagnemma K, Kang S, Shibly H, et al. Online terrain parameter estimation for wheeled mobile robots with application to planetary rovers[J]. IEEE Transactions on Robotics, 2004, 20(5):921927. 